\title{
Functional analysis of fengycin synthetase FenD
}

\author{
Tsuey-Pin Lin ${ }^{\mathrm{a}}$, Chyi-Liang Chen ${ }^{\mathrm{b}}$, Hui-Chuan Fu ${ }^{\mathrm{a}}$, Cheng-Yeu Wu ${ }^{\mathrm{b}}$, Guang-Huey Lin ${ }^{\mathrm{c}}$, \\ Shih-Hao Huang ${ }^{b}$, Li-Kwan Chang ${ }^{d}$, Shih-Tung Liu ${ }^{\text {b,* }}$ \\ ${ }^{a}$ Department of Health and Nutrition and Department of Biotechnology, Chia Nan University of Pharmacy and Science, Tainan, 701, Taiwan \\ ${ }^{\mathrm{b}}$ Molecular Genetics Laboratory, Department of Microbiology and Immunology, Chang Gung University, Kwei-Shan, 333, Taiwan \\ ${ }^{c}$ Microbial Genetics Laboratory, Institute of Microbiology Immunology and Molecular Medicine, Tzu-Chi University, Hualien, 970, Taiwan \\ ${ }^{\mathrm{d}}$ Faculty of Biological Medicine and Environmental Biology, Kaohsiung Medical University, Kaohsiung, 807, Taiwan
}

Received 18 September 2004; received in revised form 27 November 2004; accepted 15 February 2005

Available online 2 March 2005

\begin{abstract}
Fengycin is a cyclic lipopeptidic antibiotic produced nonribosomally by Bacillus subtilis. A fengycin synthetase mutant of B. subtilis F293 was generated with Tn917lux, which contains a transposon inserted in a 7716-bp gene, fenD. The mutation can be genetically complemented by transforming a plasmid carrying a wild-type fen $D$, confirming the participation of the gene in fengycin synthesis. Sequencing and biochemical analysis reveal that this gene encodes an enzyme that includes two amino acid-activating modules, FenD1 and FenD2, which activate L-Tyr and L-Thr, the third and the fourth amino acids in fengycin, respectively.

(C) 2005 Elsevier B.V. All rights reserved.
\end{abstract}

Keywords: Fengycin; Peptide synthetase; Nonribosomal peptide synthesis

\section{Introduction}

Fengycin is an antifungal antibiotic that comprises a sequence of fatty acids $\cdot \mathrm{L}-\mathrm{Glu} \cdot \mathrm{D}-\mathrm{Orn} \cdot \mathrm{L}-\mathrm{Tyr} \cdot \mathrm{D}-$ allo $\mathrm{Thr} \cdot \mathrm{L}-\mathrm{Glu} \cdot \mathrm{D}-\mathrm{Ala} / \mathrm{D}-\mathrm{Val} \cdot \mathrm{L}-\mathrm{Pro} \cdot \mathrm{L}-\mathrm{Glu} \cdot \mathrm{D}-\mathrm{Tyr} \cdot \mathrm{L}-\mathrm{Ile}$ with a lactone bond that connects L-Tyr to L-Ile [1]. Our earlier studies characterized three fengycin synthetase genes, fen $C, f e n E$ and $f e n B$, and determined the biological functions of the enzymes encoded by these genes [2-4]. As is generally known, peptide synthetases, including fengycin synthetases, generally contain one to several modules, each of which has several domains with functions required for nonribosomal peptide synthesis [57]. For instance, a peptide synthetase module typically contains an adenylation domain, which has ATPase activity and can adenylate a specific amino acid [8]. Upstream of the adenylation domain, a peptide synthetase also contains

\footnotetext{
* Corresponding author. Tel.: +886 32118292.

E-mail address: cgliu@mail.cgu.edu.tw (S.-T. Liu).
}

a condensation domain of approximately 450 amino acids, which is required to form a peptide bond between the two amino acids activated by the two adjacent modules [9]. Meanwhile, a peptide synthetase module contains a thiolation domain of about 100 amino acids downstream of an adenylation domain. After an adenylation domain has adenylated an amino acid, this adenylated amino acid is transferred and covalently linked to the cofactor, 4'phosphopantetheine, which binds to the thiolation domain, to complete the amino acid activation process $[10,11]$. In the C-terminal region, peptide synthetases normally contain an epimerization domain, which converts an L-amino acid to a D-amino acid during peptide synthesis [12], explaining why peptides synthesized nonribosomally often include D-amino acids. Finally, the module that terminates the peptide synthesis, rather than having an epimerase domain, contains a thioesterase domain in the C-terminal region to terminate peptide synthesis [13]. This study identifies a fengycin synthetase gene, $f e n D$, which encodes a fengycin synthetase that activates the third and the fourth amino acids of fengycin. 


\section{Materials and methods}

\subsection{Bacterial strains and plasmid}

Escherichia coli BL-SI (Invitrogen) was used as a host to express the proteins that contain the adenylation domains of FenD. Plasmids pDONR201 and pDEST17 (Invitrogen) were used as cloning vectors. Bacillus subtilis F29-3 is a wild-type strain that produces fengycin. FX10 is a mutant strain of B. subtilis F29-3, which contains a Tn917lux insertion in $f e n D$.

\subsection{Isolating fengycin synthesis mutants}

B. subtilis F29-3 was transformed with pD917lux [7] with a protoplast transformation method [14]. Transformants were selected on SMM-LBV agar that contained $5 \mu \mathrm{g} /$ $\mathrm{ml}$ of chloramphenicol. The transformants were subsequently cultured in LB broth that contained $0.1 \mu \mathrm{g} / \mathrm{ml}$ of erythromycin to induce transposition. Following induction, pD917lux was cured from the cell by overnight incubation at $45{ }^{\circ} \mathrm{C}$ according to a method of Youngman et al. [15]. Colonies that were resistant to chloramphenicol but sensitive to erythromycin were selected. Finally, fengycin synthesis mutants, which cannot inhibit the germination of Paecilomyces variotii spores, were identified by spotting the mutant cells on an nHA-spore plate [16]. DNA fragments adjacent to the transposon were subcloned and sequenced as described by Youngman et al. [17].

\subsection{Complementation and bioassay}

A DNA fragment that contains full-length $f e n D$ was isolated from pFD200 by NheI and XbaI digestion. The fragment was then ligated with an NheI fragment from pD917lux, which contains the pE194 replicon and an erythromycin-resistance gene. The resulting plasmid, pFD210, was transformed into the FX10 mutant of $B$. subtilis F29-3 [7]. The transformants were selected on SMM-LBV agar that contained $5 \mu \mathrm{g} / \mathrm{ml}$ of chloramphenicol and $7 \mu \mathrm{g} / \mathrm{ml}$ of erythromycin. The transformants were then subcultured in LB broth overnight. The culture $(5 \mu \mathrm{l})$ was spotted on an nHA-spore plate to examine the anti-fungal activity exhibited by the cells [16].

\subsection{Cloning FenD adenylation domains}

A DNA fragment that encodes the adenylation domain of FenD1 (AD-FenD1) was amplified using primers attB1fenD $1 \mathrm{~N}(\mathrm{~A})$ (5'-AAAAAGCAGGCTGCATCAACATTGTGCCTACT-3') and attB2-fenD1C(A) (5'AGAAAAGCTGGGTGTGCCAGTTCCGTTTCCATT-3'). A DNA fragment that encodes the adenylation domain of FenD2 (AD-FenD2) was amplified using primers attB1fenD2N(A) (5'-AAAAAGCAGGCTTAATGCAATTGCTATCTGCTCCAG) and attB2-fenD2C(A) (5'-
AGAAAAGCTGGGTGTTCTAGGGCTGATATCATTCTC). A DNA fragment that encodes the condensation, adenylation and thiolation domains of FenD1 (CATDFenD1), which includes a sequence from amino acids 1 to 1036 in FenD, was amplified with primers attB1-fenDN (5'AAAAAGCAGGCTATGACGCAAGCAACAGATCC) and attB2-fenD1C (5'-AGAAAAGCTGGGTTCACAGCGGATAAACATCTCTAAA). PCR was first performed by heating the reaction mixture at $95{ }^{\circ} \mathrm{C}$ for $2 \mathrm{~min}$, followed by ten cycles of $94{ }^{\circ} \mathrm{C}$ for $30 \mathrm{~s}, 50{ }^{\circ} \mathrm{C}$ for $30 \mathrm{~s}$ and $68{ }^{\circ} \mathrm{C}$ for 3 min, using pFC6A5 [2] as a template. Two adaptor primers, attB1 (5'-GGGGACAAGTTTGTACAAAAAAGCAGGCT) and attB2 (5'-GGGGACCACTTTGTACAAGAAAGCTGGGT), were subsequently added to the reaction mixture, which was incubated at $95{ }^{\circ} \mathrm{C}$ for $1 \mathrm{~min}$, before undergoing five cycles of PCR at $94{ }^{\circ} \mathrm{C}$ for $15 \mathrm{~s}, 45$ ${ }^{\circ} \mathrm{C}$ for $30 \mathrm{~s}, 68{ }^{\circ} \mathrm{C}$ for $3 \mathrm{~min}$ and 20 cycles at $94{ }^{\circ} \mathrm{C}$ for $15 \mathrm{~s}$, $55{ }^{\circ} \mathrm{C}$ for $30 \mathrm{~s}$ and $68{ }^{\circ} \mathrm{C}$ for $3 \mathrm{~min}$. Finally, the PCR products were inserted into pDONR201 and pDEST17 using a $\lambda$ insertion kit (Invitrogen).

\subsection{Purifying CAT-FenD1 and AD-FenD2}

Bacteria (1 1) were cultured to the mid-log phase in an $\mathrm{NaCl}$-free LB broth. The expression of recombinant proteins was induced by adding $\mathrm{NaCl}$ to the culture medium to a final concentration of $300 \mathrm{mM}$. Cells were harvested $3 \mathrm{~h}$ after induction. The pellet was suspended in $50 \mathrm{ml}$ of a binding buffer that contained $10 \mathrm{mM}$ imidazole, $300 \mathrm{mM}$ $\mathrm{NaCl}$, and $50 \mathrm{mM} \mathrm{NaH} \mathrm{PO}_{4}$ at $\mathrm{pH}$ 8.0. Cell lysate was prepared by sonication according to a method described elsewhere [2]. His-tagged recombinant proteins were finally purified with an Ni-NTA column (Qiagen) [2].

\subsection{ATP-PPi exchange assay}

The amino acid activated by CAT-FenD1 and AD-FenD2 was determined by an ATP-PPi exchange assay as described elsewhere [18]. Each reaction mixture contained $1 \mu \mathrm{Ci}$ of $\left[{ }^{32} \mathrm{P}\right]$ tetrasodium pyrophosphate $(3.03 \mathrm{Ci} / \mathrm{mmol} \mathrm{NEN}$, Boston, MA), $2 \mathrm{mM}$ amino acid, $20 \mathrm{mM}$ Hepes-MES at $\mathrm{pH}$ 6.5, with $33 \mu \mathrm{g}$ of CAT-FenD1 or $100 \mu \mathrm{g}$ of AD-FenD2.

\section{Results and discussion}

\subsection{Inserting Tn917lux into fenD}

B. subtilis F29-3 was mutagenized using Tn917lux. Colonies that contained a Tn917lux insertion were spotted on nHA-spore plates to isolate the mutants that were defective in fengycin synthesis. Fengycin is the only antibiotic produced by $B$. subtilis F29-3 that inhibits the growth of filamentous fungi [16], therefore, mutants that cannot inhibit the growth of $P$. variotii likely have a Tn917lux insertion into a gene that is involved in fengycin 


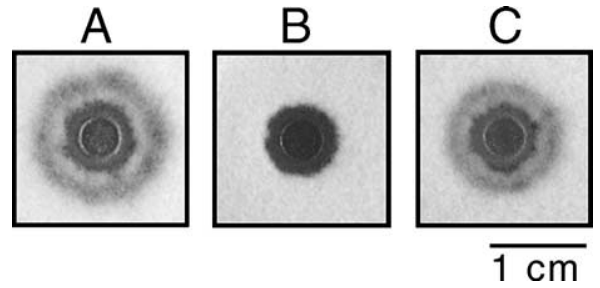

Fig. 1. Production of fengycin by $B$. subtilis F9-3 and its mutant FX10. $B$. subtilis F29-3 (A), the FX10 mutant (B), and the mutant strain that contains pFD200 (C) were spotted on a nHA-spore plate to assay their capacity to generate fengycin. The zone exhibited by FX10 (B) was attributable to competitive growth between bacteria and fungi.

synthesis. Among about 3000 colonies examined, 72 could not synthesize fengycin and were unable to inhibit the growth of $P$. variotii on bioassay plates. The insertion of a transposon into these mutants was subsequently analyzed by subcloning and sequencing. Of these mutants, FX10 contained a Tn917lux inserted into fenD. This gene was positioned 29 nucleotides downstream of a previously characterized fengycin synthetase gene, $f e n C$ [2]. Sequencing analysis demonstrated that Tn917lux was inserted 3210 nucleotides downstream of the translation initiation codon of fenD. On a bioassay plate, wild-type B. subtilis F29-3 produced an inhibition zone surrounded by a white lawn of fungal hyphae (Fig. 1A). However, the FX10 mutant did not establish a similar inhibition zone (Fig. 1B). Although FX10 seemed to yield a smaller zone, this zone actually contained an inner circle in which the bacterial culture was originally applied and an outer ring where agar was covered by bacteria. Such type of inhibition is attributable to competitive growth between bacteria and fungi, rather than from the suppression of fungal growth by an antibiotic produced

Table 1

Comparison of conserved sequences in peptide synthetases and FenD

\begin{tabular}{|c|c|c|c|c|c|}
\hline Domain & Core & Sequence conserved in peptide synthetases ${ }^{\mathrm{a}}$ & Sequence in FenD & Module in $f e n D$ & Positions $^{\mathrm{b}}$ \\
\hline \multirow[t]{12}{*}{ Condensation } & \multirow[t]{2}{*}{$\mathrm{C} 1$} & \multirow[t]{2}{*}{ SxAQxR(L/M)(W/Y)xL } & SYMQEGMIFH & D1 & $14-23$ \\
\hline & & & SFSQKRLYAL & D2 & $1059-1068$ \\
\hline & \multirow[t]{2}{*}{$\mathrm{C} 2$} & \multirow[t]{2}{*}{ RHExLRTxF } & RYDIFRTIF & D1 & $60-68$ \\
\hline & & & RHESLRTAF & D2 & $1105-1113$ \\
\hline & \multirow[t]{2}{*}{$\mathrm{C} 3$} & \multirow[t]{2}{*}{ MHHxISDG(W/V)S } & HHHIIMDG & D1 & $145-154$ \\
\hline & & & MHHIIADGIS & D2 & $1180-1189$ \\
\hline & \multirow[t]{2}{*}{$\mathrm{C} 4$} & \multirow[t]{2}{*}{$\mathrm{YxD}(\mathrm{F} / \mathrm{Y}) \mathrm{AVW}$} & YSRYIEW & D1 & $183-189$ \\
\hline & & & YKDFAVW & D2 & $1213-1219$ \\
\hline & \multirow[t]{2}{*}{$\mathrm{C} 5$} & \multirow[t]{2}{*}{$(\mathrm{I} / \mathrm{V}) \mathrm{GxFVNT}(\mathrm{Q} / \mathrm{L})(\mathrm{C} / \mathrm{A}) \mathrm{xR}$} & VGLFINTVPVR & D1 & $296-306$ \\
\hline & & & IGMFVNTLAMR & D2 & $1328-1338$ \\
\hline & C6 & $(\mathrm{H} / \mathrm{N}) \mathrm{QD}(\mathrm{Y} / \mathrm{V}) \mathrm{PFE}$ & HQDYPFE & D2 & $1364-1370$ \\
\hline & $\mathrm{C} 7$ & RDxSRNPL & EVNRNPL & D2 & $1381-1387$ \\
\hline \multirow[t]{20}{*}{ Adenylation } & \multirow[t]{2}{*}{ A1 } & \multirow[t]{2}{*}{$\mathrm{L}(\mathrm{T} / \mathrm{S}) \mathrm{YxEL}$} & RTYRQL & D1 & $495-500$ \\
\hline & & & LTYRDL & D2 & $1526-1531$ \\
\hline & \multirow[t]{2}{*}{$\mathrm{A} 2$} & \multirow[t]{2}{*}{ LKAGxAYL(V/L)P(L/I)D } & WKAGGAYLPLD & D1 & $540-550$ \\
\hline & & & LKAGGAYLPID & D2 & $1570-1581$ \\
\hline & \multirow[t]{2}{*}{ A3 } & \multirow[t]{2}{*}{ LAYxxYTSG(S/T)TGxPKG } & LAYLIYTSGTTGRPKG & D1 & $609-624$ \\
\hline & & & PAYVIYTSGTTGQPKG & D2 & $1642-1657$ \\
\hline & \multirow[t]{2}{*}{ A4 } & \multirow[t]{2}{*}{ FDxS } & FDGC & D1 & $659-662$ \\
\hline & & & FDFG & D2 & $1692-1695$ \\
\hline & \multirow[t]{2}{*}{ A5 } & \multirow[t]{2}{*}{ NxYGPTE } & NEYGPTE & D1 & $753-759$ \\
\hline & & & NMYGITE & D2 & $1787-1789$ \\
\hline & \multirow[t]{2}{*}{ A6 } & \multirow[t]{2}{*}{ GELxIxGxG(V/L)ARGYL } & GELCISGAGLARGYY & D1 & $802-816$ \\
\hline & & & GELYIGGEGVARGYL & D2 & $1838-1853$ \\
\hline & \multirow[t]{2}{*}{ A7 } & $\mathrm{Y}(\mathrm{R} / \mathrm{K}) \mathrm{TGDL}$ & YRTGDA & D1 & $838-843$ \\
\hline & & & YRTGDL & D2 & $1875-1880$ \\
\hline & A8 & GRxDxQVKIRGxRIELGEIE & GRFDDQVKIRGYRIELSEIE & D1 & $856-875$ \\
\hline & & & GRIDDQVKVRGHRIELGEIQ & D2 & 1893-1912 \\
\hline & A9 & LPxYM(I/V)P & LPSYMIP & D1 & 924-930 \\
\hline & & & LPDFMVP & D2 & $1964-1970$ \\
\hline & A10 & NGK(V/L)DR & SGKLDR & D1 & 944-949 \\
\hline & & & NGKLDQ & D2 & 1984-1989 \\
\hline Thiolation & $\mathrm{T}$ & DxFFxxLGG(H/D)S(L/I) & DDFFQLGGQSL & D1 & $991-1001$ \\
\hline & & & ANFFELGGDSI & D2 & 2032-2042 \\
\hline Epimerization & E1 & PIQxWF & PVQQWF & D2 & 2095-2101 \\
\hline & E2 & HHxISDG(W/V)S & HHLVIDAVS & D2 & $2215-2223$ \\
\hline & E3 & DxLLxAxG & DILLTALG & D2 & $2349-2356$ \\
\hline & E4 & EGHGRE & EGHGRE & D2 & $2373-2378$ \\
\hline & E5 & RTVGWFTxxYP(Y/V)PFE & RTVGWFTSIYPVW & D2 & 2389-2401 \\
\hline & E6 & PxxGxGYG & PHKGAGYG & D2 & $2430-2437$ \\
\hline & E7 & FNYLG(Q/R) & FNYLGQ & D2 & $2457-2462$ \\
\hline
\end{tabular}

${ }^{\mathrm{a}}$ From reference [9].

b Amino acid positions in FenD. 
by bacteria since similar zones were also observed when an E. coli culture was applied to the plate. More importantly, the capacity to produce fengycin was restored when the mutant was transformed with pFD210 (Fig. 1C), indicating that the mutation can be genetically complemented and fenD participates in fengycin synthesis.

\subsection{Sequence of FenD}

In mutant FX10, Tn917lux was inserted in fenD, which is 7716 nucleotides long (GenBank accession number: AJ011849) and preceded by a Shine-Dalgarno sequence, 5'-TGGA, five nucleotides upstream of the initiation codon of the gene. Sequence analysis predicts that FenD has a molecular mass of $287 \mathrm{kDa}$ and contains two sets of motifs that are commonly found in the condensation, adenylation, and thiolation domains in peptide synthetases (Table 1) [9], indicating that FenD contains two amino acid activation modules - FenD1 and FenD2. Therefore, FenD probably activates two amino acids during fengycin synthesis. Meanwhile, FenD1 includes a condensation domain between amino acids 1 and 444, an adenylation domain between amino acids 445 and 957 , and a thiolation domain between amino acids 976 and 1036. These domains in FenD2 are between amino acids 1037 and 1474, 1475 and 2016, and 2017 and 2076, respectively. Additionally, FenD has a C-terminal region that is 496 amino acids long, which includes seven conserved motifs that are typically present in an epimerase domain (Table 1) [9], indicating that this region contains an epimerase domain and the amino acid activated by FenD2 is epimerized during fengycin synthesis. Furthermore, among the seven conserved motifs identified in the condensation domain in FenD2, C6 and C7 are absent from the corresponding domain in FenD1 (Table 1). These two motifs were also found to be absent from the first module of a peptide synthetase in other peptide synthetases, including those involved in fengycin, surfactin and tyrocidine synthesis $[2,4,19,20]$. The functions of these two motifs are unknown.

\subsection{Expressing and purifying the proteins that contain the amino acid adenylation domains of FenD}

A DNA fragment that encodes the region between amino acids 444 and 975 of FenD was amplified by PCR and cloned into pDEST17 to express a recombinant protein that contains the adenylation domain of FenD1 (AD-FenD1) in E. coli BL-SI. Following $\mathrm{NaCl}$ treatment to induce the expression of the recombinant protein, a cell lysate was prepared from the cells. SDS-polyacrylamide gel electrophoresis revealed that AD-FenD1 was present in the pellet fraction, indicating that the protein was insoluble and could not be used in enzymatic analysis. Meanwhile, a recombinant protein that contains the condensation, adenylation, and thiolation domains of FenD1 (CATD-FenD1) was expressed in E. coli BL-SI (Fig. 2, lanes 1 and 2) and was present in
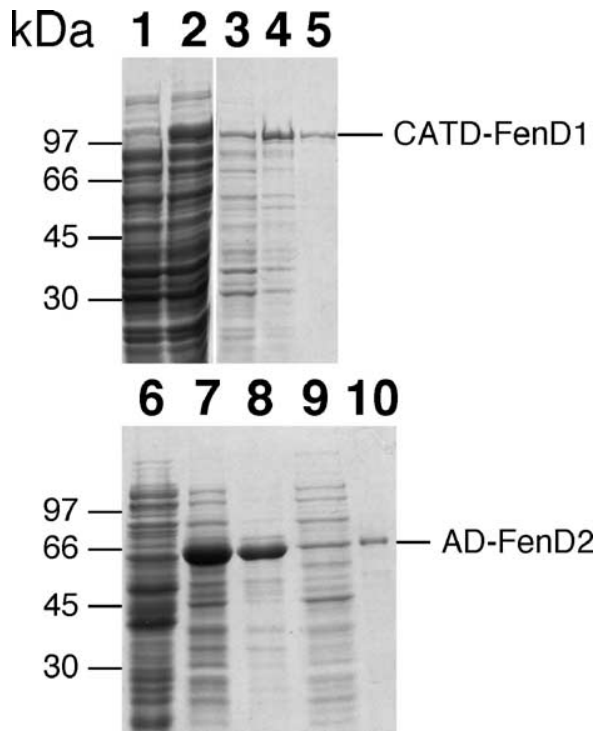

Fig. 2. Expressing and purifying recombinant FenD adenylation domains. Cell lysates were prepared from $E$. coli BL-SI expressing CATD-FenD1 (lanes 1 to 5) and AD-FenD2 (lanes 6 to 10). Cells were lysed before (lanes 1 and 6) and after (lanes 2 and 7) $\mathrm{NaCl}$ induction. After induction, cells were homogenized by sonication and lysates were centrifuged to separate the supernatant (lanes 4 and 9) and the pellet (lanes 3 and 8) fractions. Recombinant protein in the soluble fraction was finally purified using an Ni-NTA column (lanes 5 and 10).

both the pellet and the supernatant fraction of the cell lysate (Fig. 2, lanes 3 and 4). Furthermore, the adenylation domain of FenD2 (AD-FenD2), between amino acids 1569 and 2108 in FenD, was also present in the pellet and the supernatant fractions of the cell lysate (Fig. 2, lanes 8 and 9). Hence, CATD-FenD1 and AD-FenD2 were purified from the cell lysate with an Ni-NTA column (Fig. 2, lanes 5 and 10).

\subsection{Amino acids activated by FenD}

The amino acids activated by the adenylation domains of FenD were first analyzed using the eight amino acids in the fengycin molecule as the substrate. ATP-PPi exchange assay demonstrated that adding L-Tyr to a reaction mixture that contained $33 \mu \mathrm{g}$ of CATD-FenD1 yielded the highest exchange activity, indicating that FenD1 activates L-Tyr, the third amino acid in the fengycin molecule (Fig. 3A). Meanwhile, adding the 13 common amino acids that were absent from the fengycin molecule yielded only background counts (data not shown), indicating that L-Tyr is the only amino acid to be activated by AD-FenD1. According to Marahiel et al. [21], a peptide synthetase can use D-amino acids as a substrate in vitro if the condensation domain of the enzyme remains intact. However, in the case of CATDFenD1, this enzyme exhibited an exchange activity of about tenfold above the background when D-Tyr was used as the substrate, but the activity was substantially lower than that exhibited by the enzyme when L-Tyr was used as the substrate (Fig. 3A), suggesting that FenD1 does not 

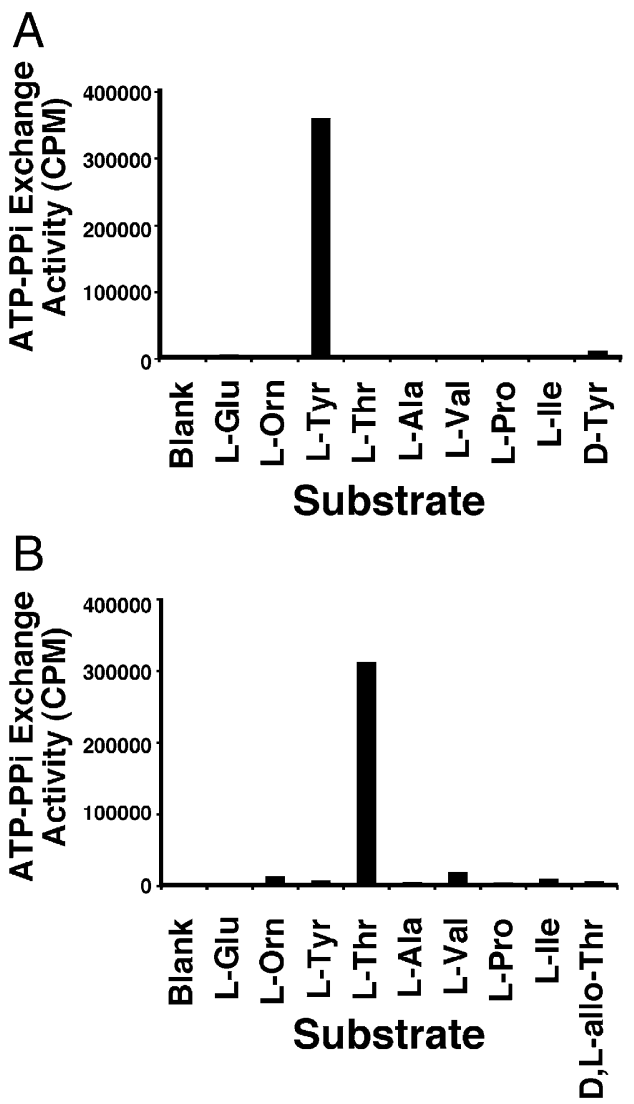

Fig. 3. ATP-PPi exchange activities of CATD-FenD1 (A) and AD-FenD2 (B).

efficiently use D-Tyr as a substrate. In the case of ADFenD2, adding L-Thr to a reaction mixture that contained $100 \mu \mathrm{g}$ of AD-FenD2 yielded the highest exchange activity (Fig. 3B). Neither the other seven amino acids in the fengycin molecule (Fig. 3B) nor the other 13 common amino acids yielded a high level of exchange activity, indicating that $\mathrm{AD}-\mathrm{FenD} 2$ activates only $\mathrm{L}-\mathrm{Thr}$, which is then converted into D-allo-Thr, the fourth amino acid in the fengycin molecule. Indeed, AD-FenD2 does not use D-Thr as a substrate $[21,22]$. Moreover, D-allo-Thr can only be generated following epimerization.

\subsection{Biochemical characterization of CATD-FenD1 and AD-FenD2}

The enzymatic activity of CATD-FenD1 and AD-FenD2 was investigated under various reaction conditions. These two recombinant proteins have an optimal $\mathrm{pH}$ between 6.5 and 8.0, which resembles the optimal $\mathrm{pH}$ range of FenE1 and FenE2 [4] but is much higher than those of FenB, FenC1, and FenC2, which have an optimal $\mathrm{pH}$ of 4.5-5.0 $[2,3]$. This may indicate that fengycin synthetases function in various $\mathrm{pH}$ environments in the cells. Meanwhile, CATDFenC1 and AD-FenD2 also have an optimal activity at 25 ${ }^{\circ} \mathrm{C}$, which is consistent with the optimal temperature for the adenylate activation reactions associated with the activation domains of FenC and FenB [2,3]. At optimal $\mathrm{pH}$ and temperature, CATD-FenD1 has a KM value of $0.051 \mathrm{mM}$ and a kcat value of $26 \mathrm{~s}^{-1}$ for L-Tyr; AD-FenD2 has a KM value of $0.058 \mathrm{mM}$ and a kcat value of $2.65 \mathrm{~s}^{-1}$ for L-Thr.

Five peptide synthetases participate in fengycin synthesis $[2-4,6]$. Experimental data currently available indicate that the amino acid sequence in fengycin is colinear with the genes in the fengycin synthetase operon [2-4]. As is generally known, B. subtilis 168 contains a pps operon, which is involved in plipastatin synthesis. This operon comprises five genes that encode enzymes with a sequence that is about $72-80 \%$ identical to the sequence of fengycin synthetases [2]. Steller et al. [23] observed that B. subtilis b213 produces a fengycin-like antibiotic, which is probably plipastatin, because the eighth amino acid of the antibiotic is glutamine. They purified three peptide synthetases, which have a molecular mass of $286 \mathrm{kDa}$, as a mixture. Biochemical analysis indicated that these enzymes can activate the six N-terminal amino acids of plipastatin. These findings are consistent with the results concerning the molecular mass and biochemical functions of FenD. Later, Tsuge et al. [24] renamed the pps operon pli to reflect the fact that the operon produces plipastatin rather than fengycin. They also determined that the expression of the pli operon depends on $\operatorname{deg} Q$. These findings demonstrate that fen and pli operons are evolutionarily conserved by Bacillus spp.; the production of either antibiotic may help the organisms to survive in nature. The results herein constitute a valuable reference for future investigations that involve nonribosomal peptide synthesis and provide further insight into the synthesis of fengycin by B. subtilis F29-3.

\section{Acknowledgements}

This work was supported by grants from the Chang-gung Memorial Hospital (CMRP1211) and from the National Science Council of the Republic of China (NSC92-2320-B182-044 and NSC90-2320-B-041-014).

\section{References}

[1] U. Koch, Fengycin: Strukturaufklärung eines mikroheterogenen Lipopeptidantibiotikums. Dissertation, zur Erlangung des Grades eines Doktors, der Naturwissenschaften. Eberhard-Karls-Universität zu Tübingen, Tübingen, Germany, 1988.

[2] T.P. Lin, C.L. Chen, L.K. Chang, J.S. Tschen, S.T. Liu, Functional and transcriptional analyses of a fengycin synthetase gene, fenC, from Bacillus subtilis, J. Bacteriol. 181 (1999) 5060-5067.

[3] G.H. Lin, C.L. Chen, J.S. Tschen, S.S. Tsay, Y.S. Chang, S.T. Liu, Molecular cloning and characterization of fengycin synthetase gene fenB from Bacillus subtilis, J. Bacteriol. 180 (1998) 1338-1341.

[4] H.Y. Shu, G.H. Lin, Y.C. Wu, J.S. Tschen, S.T. Liu, Amino acids activated by fengycin synthetase FenE, Biochem. Biophys. Res. Commun. 292 (2002) 789-793.

[5] H. Kleinkauf, H. Von Döhren, A nonribosomal system of peptide biosynthesis, Eur. J. Biochem. 236 (1996) 335-351.

[6] C.L. Chen, L.K. Chang, Y.S. Chang, S.T. Liu, J.S. Tschen, Trans- 
poson mutagenesis and cloning of the genes encoding the enzymes of fengycin biosynthesis in Bacillus subtilis, Mol. Gen. Genet. 248 (1995) $121-125$

[7] L.K. Chang, C.L. Chen, Y.S. Chang, J.S. Tschen, Y.M. Chen, S.T. Liu, Construction of Tn917ac1, a transposon useful for mutagenesis and cloning of Bacillus subtilis genes, Gene 150 (1994) 129-134.

[8] T. Stachelhaus, M.A. Marahiel, Modular structure of peptide synthetases revealed by dissection of the multifunctional enzyme GrsA, J. Biol. Chem. 270 (1995) 6163-6169.

[9] M.A. Marahiel, T. Stachelhaus, H.D. Mootz, Modular peptide synthetases involved in nonribosomal peptide synthesis, Chem. Rev. 97 (1997) 2651-2674.

[10] T. Stein, J. Vater, V. Kruft, A. Otto, B. Wittmann-Liebold, P. Franke, M. Panico, R. McDowell, H.R. Morris, The multiple carrier model of nonribosomal peptide biosynthesis at modular multienzymatic templates, J. Biol. Chem. 271 (1996) 15428-15435.

[11] M.R. Mofid, R. Finking, L.O. Essen, M.A. Marahiel, Structure-based mutational analysis of the $4^{\prime}$-phosphopantetheinyl transferases Sfp from Bacillus subtilis: carrier protein recognition and reaction mechanism, Biochemistry 43 (2004) 4128-4136.

[12] V. De Crécy-Lagard, P. Marliere, W. Saurin, Multienzymatic non ribosomal peptide biosynthesis: identification of the functional domains catalysing peptide elongation and epimerisation, C. R. Acad. Sci., Ser. III 318 (1995) 927-936.

[13] A. Schneider, M.A. Marahiel, Genetic evidence for a role of thioesterase domains, integrated in or associated with peptide synthetases, in non-ribosomal peptide biosynthesis in Bacillus subtilis, Arch. Microbiol. 169 (1998) 404-410.

[14] T. Imanaka, M. Fujii, I. Aramori, S. Aiba, Transformation of Bacillus stearothermophilus with plasmid DNA and characterization of shuttle vector plasmids between Bacillus stearothermophilus and Bacillus subtilis, J. Bacteriol. 149 (1982) 824-830.

[15] P.J. Youngman, J.B. Perkins, R. Losick, Genetic transposition and insertional mutagenesis in Bacillus subtilis with Streptococcus faecalis transposon Tn917, Proc. Natl. Acad. Sci. U. S. A. 80 (1983) 2305-2309.

[16] W. Loeffler, J.S.M. Tschen, N. Vanittanakom, M. Kugler, E. Knorpp, T.G. Wu, Antifungal effects of bacilysin and fengycin from Bacillus subtilis F29-3. A comparison with activities of other Bacillus antibiotics, J. Phytopathol. 115 (1986) 204-213.

[17] P. Youngman, J.B. Perkins, R. Losick, Construction of a cloning site near one end of Tn917 into which foreign DNA may be inserted without affecting transposition in Bacillus subtilis or expression of the transposon-borne erm gene, Plasmid 12 (1984) 1-9.

[18] S.G. Lee, F. Lipmann, Tyrocidine synthetase system, Methods Enzymol. 43 (1975) 585-602.

[19] P. Cosmina, F. Rodriguez, F. de Ferra, G. Grandi, M. Perego, G. Venema, D. van Sinderen, Sequence and analysis of the genetic locus responsible for surfactin synthesis in Bacillus subtilis, Mol. Microbiol. 8 (1993) $821-831$

[20] H.D. Mootz, M.A. Marahiel, The tyrocidine biosynthesis operon of Bacillus brevis: complete nucleotide sequence and biochemical characterization of functional internal adenylation domains, J. Bacteriol. 179 (1997) 6843-6850.

[21] U. Linne, M.A. Marahiel, Control of directionality in nonribosomal peptide synthesis: role of the condensation domain in preventing misinitiation and timing of epimerization, Biochemistry 39 (2000) 10439-10447.

[22] S. Doekel, M.A. Marahiel, Dipeptide formation on engineered hybrid peptide synthetases, Chem. Biol. 7 (2000) 373-384.

[23] S. Steller, D. Vollenbroich, F. Leenders, T. Stein, B. Conrad, J. Hofemeister, P. Jacques, P. Thonart, J. Vater, Structural and functional organization of the fengycin synthetase multienzyme system from Bacillus subtilis b213 and A1/3, Chem. Biol. 6 (1999) 31-41.

[24] K. Tsuge, T. Ano, M. Hirai, Y. Nakamura, M. Shoda, The genes $\operatorname{deg} Q$, pps, and lpa-8 (sfp) are responsible for conversion of Bacillus subtilis 168 to plipastatin production, Antimicrob. Agents Chemother. 43 (1999) 2183-2192. 